\title{
PRINCIPIOS BÁSICOS Y ALCANCES METODOLÓGICOS DE LAS EVALUACIONES ECONÓMICAS EN SALUD
}

\author{
Cesar Loza ${ }^{1,2, a}$, Manuel Castillo-Portilla ${ }^{1, b}, J^{\prime}$ sé Luis Rojas ${ }^{1, b}$, Leandro Huayanay ${ }^{1,2,3, c}$
}

\begin{abstract}
RESUMEN
La economía de la salud es un instrumento indispensable para la gestión sanitaria y las evaluaciones económicas, se pueden considerar como la herramienta que asiste a la toma de decisiones para asignar recursos en el área de la salud. Hoy en día, su uso es creciente en todas las regiones del mundo y fomenta la toma de decisiones basadas en la evidencia, buscando alternativas eficientes y racionales dentro del conjunto de actividades de los servicios. En esta revisión se efecturá una visión general y se delinea los tipos básicos de evaluación económica, sobre todo de las Evaluaciones Económicas (EE) completas. Así mismo se revisará los conceptos más relevantes sobre las perspectivas desde las que se pueden realizar las EE, los tipos de costos, el horizonte temporal, los descuentos, la evaluación de la incertidumbre y las reglas de decisión. Finalmente, se describirán conceptos sobre la transferibilidad y la generalización de las Evaluaciones Económicas en salud.
\end{abstract}

Palabras clave: Economía de la salud; Costos de atención médica; Costos y análisis de costo; Análisis costo-eficiencia; Análisis costo-beneficio (fuente: DeCS BIREME).

\section{BASIC PRINCIPLES AND METHODOLOGICAL CONSIDERATIONS OF HEALTH ECONOMIC EVALUATIONS}

\begin{abstract}
Health Economics is an essential instrument for health management, and economic evaluations can be considered as tools assisting the decision-making process for the allocation of resources in health. Currently, economic evaluations are increasingly being used worldwide, thus encouraging evidence-based decision-making and seeking efficient and rational alternatives within the framework of health services activities. In this review, we present an overview and define the basic types of economic evaluations, with emphasis on complete Economic Evaluations (EE). In addition, we review key concepts regarding the perspectives from which EE can be conducted, the types of costs that can be considered, the time horizon, discounting, assessment of uncertainty and decision rules. Finally, we describe concepts about the extrapolation and spread of economic evaluations in health.
\end{abstract}

Keywords: Health economics; Health care costs; Costs and cost analysis; Cost efficiency analysis; Cost-benefit analysis (source: MeSH NLM).

\section{CONCEPTOS GENERALES SOBRE LAS EVALUACIONES ECONÓMICAS}

El desarrollo económico de un país es un factor determinante en el estado de salud de su población que, a su vez, es determinante del desarrollo económico. La unión del "enfoque de producción" en economía y las "premisas de bienestar social" en salud, han dado lugar a la "economía de la salud". Estos conceptos se han incorporado en el desempeño médico y en la toma de decisiones basadas en evidencias, en especial si consideramos el beneficio esperado en el paciente o en la población. Actualmente, son varias las publicaciones sobre el uso racional de los recursos en la toma de decisiones médicas; por lo tanto, los profesionales de la salud tienen que adquirir conocimientos básicos de la materia, conocer el lenguaje, sus conceptos y sus aplicaciones ${ }^{(1)}$.

La economía de la salud es, en principio, la economía aplicada a la atención sanitaria y se usa, sobre todo para

\footnotetext{
Unidad de Epidemiología Clínica. Facultad de Medicina Alberto Hurtado, Universidad Peruana Cayetano Heredia. Lima, Perú.

Hospital Nacional Cayetano Heredia. Lima, Perú.

3 Dirección General de Insumos y Drogas (DIGEMID), Ministerio de Salud del Perú. Lima, Perú.

a Médico Nefrólogo, Epidemiólogo; ${ }^{b}$ Médico Cirujano; ${ }^{c}$ Médico Internista, Epidemiólogo
}

Recibido: 16-06-11 Aprobado: 24-08-11 
ayudar a los políticos y técnicos en la toma de decisiones en salud. Asimismo, nos permite evaluar los recursos destinados a la salud frente a otros sectores económicos y como se distribuyen los recursos en salud entre diversas personas o grupos sociales (equidad) y de qué manera se utilizan los recursos de salud asignados a una persona o grupo social (eficiencia). No es posible imaginar ninguna estabilidad política o crecimiento económico sin que el bienestar se extienda a la sociedad en su conjunto, es decir, sin que las coberturas de salud y las oportunidades sociales sean iguales para todos ${ }^{(2)}$.

Un buen sistema de salud debe ser universal y de calidad, y los costos que demandan deben ser cubiertos por la sociedad. Esta triada se torna incompatible, ya que el sistema no siempre es universal por los excluidos y, si es universal no es de calidad, y menos aun sostenible financieramente. En realidad, los presupuestos de los sistemas de salud son insuficientes para cubrir todas las necesidades y la demanda es relativamente constante, por lo que las leyes de oferta y demanda no funcionan con eficiencia. Por otro lado, los mercados de salud son imperfectos, magnificándose esta característica en el mercado farmacéutico.

Convencionalmente los medicamentos se escogen para ser utilizados teniendo en cuenta la eficacia, seguridad y calidad. Australia fue el primer país que tomó en cuenta el precio de los medicamentos para reembolsos en su sistema de salud, luego diversos países desarrollados como Reino Unido, Canadá, etc., tomaron en cuenta el costo de un medicamento para su utilización, constituyendo la llamada "cuarta barrera" (3). Para considerar los aspectos económicos en la utilización de los medicamentos, se deben realizar evaluaciones económicas que determinen si el medicamento es costo-efectivo y si, por los beneficios que dicho medicamento trae al paciente, vale la pena que el sistema de salud lo financie o no.

Es errado ver solo costos para tomar decisiones en salud; para poder hacer elecciones apropiadas, el profesional requiere información esencial relativa a, por lo menos, dos características básicas de las tecnologías sanitarias: los costos y las consecuencias (o resultados) de la misma. En términos sencillos, el propósito de la Evaluación Económica (EE) en salud, es elegir entre varias opciones, la más ventajosa. En palabras del profesor Charles Normand, se debe: identificar y proporcionar aquellos servicios que tienen el mayor efecto sobre la salud ${ }^{(4)}$.

Entonces, las EE son, por un lado, una manera de valorar la labor médica y, por otro, un conjunto de instrumentos que permiten operacionalizar este enfoque. El criterio esencial parte de la verificación de que los recursos disponibles son siempre menores que las necesidades y, por lo tanto, se debe procurar su uso más racional. Finalmente, podemos definir a la economía de la salud como el instrumento indispensable para la gestión sanitaria y la asignación de recursos, que permite orientar la toma de decisiones para buscar alternativas eficientes y racionales dentro del conjunto de actividades de los servicios y las diferentes tecnologías del sistema nacional de salud ${ }^{(5)}$.

\section{TIPOS DE COSTOS}

Los tipos de costos son directos e indirectos. Los directos son todos aquellos directamente relacionados con la implementación de determinado programa o la administración de determinada intervención; se clasifican en costos directos del sistema de salud (consultas médicas, de enfermería, de drogas y otras tecnologías) y costos del paciente, de la familia (gastos por transporte, para contratar ayuda informal) y de otros sectores.

Los costos indirectos, mejor denominados costos de productividad perdida, se refieren a la valoración del tiempo que el paciente o algún miembro de la familia debe dejar de trabajar debido a la intervención considerada. En los diseños de las EE debe siempre incluirse el tipo de costo según la perspectiva asumida.

Los costos desde la perspectiva social son más completos, pues se deben incluir todos los recursos consumidos, sin importar quien los consuma, ya que todos los costos son relevantes desde la perspectiva social, como los costos por desintegración familiar ${ }^{(6,7)}$.

\section{TIPOS DE EVALUACIONES ECONÓMICAS}

Para la toma de decisiones sanitarias las dimensiones más importantes son la efectividad de los tratamientos y sus costos. La efectividad es un concepto muy amplio y contempla aspectos como la reducción de la mortalidad, incremento de la sobrevida, mejora en la calidad de vida, etc. Al referirnos a los costos, incluimos la valoración en términos monetarios de todos los recursos relevantes implicados en el uso de las tecnologías por evaluar.

Evaluaciones parciales. Si las EE solo incluyen una de las dimensiones señaladas, son llamadas EE parciales. Por ejemplo, cuando queremos comparar dos intervenciones para la misma enfermedad, podemos comparar cuánto nos cuesta cada una de ellos. En el caso de pacientes con apendicitis aguda que van a ser intervenidos de emergencia, podemos comparar el costo de la apendicectomía convencional y la apendicectomía laparoscópica. En el análisis incluiremos los costos relevantes 
Tabla 1. Clasificación de evaluaciones económicas parciales y completas

\begin{tabular}{|c|c|c|c|c|}
\hline & & \multicolumn{3}{|c|}{ ¿Considera costos y consecuencias de las alternativas seleccionadas? } \\
\hline & & \multicolumn{2}{|c|}{ NO } & \multirow[t]{2}{*}{ Sí } \\
\hline & & $\begin{array}{c}\text { Evalúa solo } \\
\text { consecuencias }\end{array}$ & Evalúa solo costos & \\
\hline \multirow[b]{2}{*}{$\begin{array}{l}\text { ¿Compara dos o } \\
\text { más alternativas? }\end{array}$} & $\mathrm{NO}$ & $\begin{array}{l}\text { Descripción de } \\
\text { resultados }\end{array}$ & $\begin{array}{l}\text { Descripción de } \\
\text { costos }\end{array}$ & Descripción de costos por resultados \\
\hline & sí & $\begin{array}{c}\text { Evaluación de la } \\
\text { eficacia o efectividad }\end{array}$ & Análisis de costos & $\begin{array}{c}\text { Evaluaciones económicas completas: } \\
\text { Minimización de costos; Costo- efectividad; } \\
\text { Costo-utilidad; Costo-beneficio. }\end{array}$ \\
\hline
\end{tabular}

Fuente: Drummond, Stoddart and Torrance, 1987.

como los honorarios médicos, el uso de la sala de operaciones, etc.

Otros calculan la carga económica de una enfermedad mediante estudios de "costo de enfermedad". Por ejemplo, Amentand y Evers ${ }^{(8)}$, estudiaron en Holanda el costo que generan enfermedades tan comunes como la dispepsia y la esquizofrenia, estimando los costos de la dispepsia en USD 785,5 millones por año y la esquizofrenia en USD 563,6 millones.

Por otro lado, podemos tener EE donde solo se evalúan los resultados clínicos (efectos), sin importar los recursos empleados. Aquí son clásicos los estudios clínicos que aportan información valiosa en cuanto a la efectividad de las diferentes intervenciones, pero no dicen nada acerca de los costos que estas intervenciones implican para el sistema de salud.

Evaluaciones completas. Son los estudios más recomendados pues incorporan las dos dimensiones, evaluando tanto los costos como las consecuencias, para ello es necesario comparar dos o más intervenciones incluyendo tanto los efectos como los costos. Este tipo de EE constituye una valiosa herramienta para la toma de decisiones, ya que brinda información que permite comparar dos o más intervenciones en sus dimensiones más relevantes (Tabla 1).

En general, solo los estudios del cuadrante inferior derecho, donde se comparan tanto costos como consecuencias de dos o más intervenciones, se aceptan como EE Completas, siendo todas las demás formas de EE Parciales.

El resultado (outcome) o efecto, constituye las consecuencias de las intervenciones y pueden ser clasificados en tres grandes grupos:

1. Efectos: cambios en el funcionamiento fisiológico, individual o social.

2. Utilidad: cambios en la cantidad y calidad de vida de los pacientes y sus familias.

\section{Beneficios: cambios en el uso de recursos.}

Estas diferencias han dado origen a las diferentes técnicas de EE y ha permitido clasificar a las EE completas.

\section{ANÁLISIS DE COSTO BENEFICIO (ACB)}

Se emplea para comparar programas con diferentes resultados, haciendo que el denominador común a comparar sea el dinero, al dar un valor monetario a los beneficios obtenidos (como días de trabajo perdidos evitados, los años de vida ganados, las complicaciones médicas evitadas, etc.).

Por ejemplo, al evaluar la posibilidad de un programa de fluoración del agua potable para prevenir la caries, los costos pueden ser los de fluoración y, el beneficio, mejorar la salud de la población, que en dinero representa lo que se ahorrará al no tener que atender a los que no desarrollarán caries (beneficio monetario). Si el ahorro es USD 1500000 y los costos USD 500 000, entonces el cociente beneficio/costo será ahorros/costos: USD $1500000 /$ USD $500000=3$. Por lo tanto, los beneficios monetarios son mayores que los costos monetarios, así el programa será rentable. La dificultad está en la valoración monetaria de los cambios en el estado de salud. En este caso, los resultados clínicos se traducen en unidades monetarias y requieren que se adjudique un valor monetario a las consecuencias. EI ACB se puede expresar como el cociente beneficio (USD)/costo (USD). Es el único tipo de evaluación económica que permite evaluar eficiencia asignativa y es el único que está incluido en la "teoría del bienestar" (Figura 1).

La ventaja del ACB es que aporta información sobre el beneficio absoluto de los programas y proporciona un valor estimado de los recursos utilizados por cada programa, comparado con los recursos que podría ahorrarse o crear. Entre las desventajas del ACB es que se requiere que la vida humana y la calidad de vida tengan que ser evaluados en unidades monetarias. Esta tarea no es ética, es difícil de asignar valores monetarios y los métodos de hacerlo son complejos. 


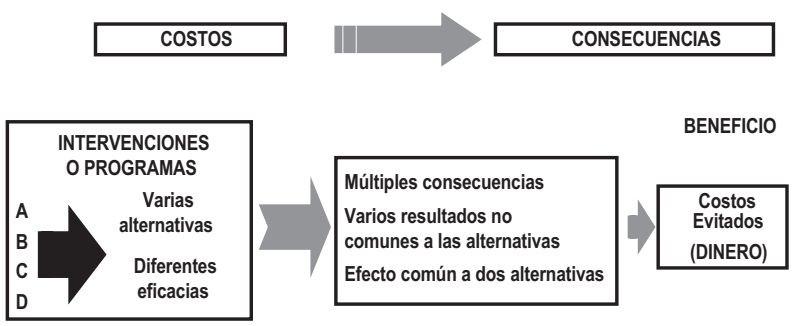

Figura 1. Esquema de los análisis costo-beneficio.

Un proyecto o política mejorará la asistencia social si el beneficio asociado con el proyecto o política excede los costos. En el caso del cociente ahorros/costos en la fluoración del agua, los ahorros son los costos evitados y los costos representan los costos de la fluoración. Los costos evitados muchas veces pueden distorsionar los resultados. En salud es frecuente este fenómeno ya que la meta primaria en muchas propuestas (medicina preventiva) es mejorar la salud y reducir los costos subsecuentes del tratamiento médico (costos evitados). En el ACB la valoración de los beneficios es un desafío, no existen precios en el mercado para los beneficios. Entonces ¿Cómo asignar valores monetarios a los resultados en salud? En realidad existen tres métodos: i) EI método del capital humano (Ej. salarios); ii) las preferencias de los pacientes y iii) la disposición a pagar (método de la preferencia revelada o willingness to pay) ${ }^{(9,10)}$.

\section{ANÁLISIS DE COSTO EFECTIVIDAD (ACE)}

En el ACE se miden los mismos resultados con diferentes alternativas. Los resultados pueden presentarse en dos formas; como un cociente de costo-efectividad, es decir el costo de una unidad adicional de un resultado de salud, o bien como incremento de dicha razón (costoefectividad incremental) ${ }^{(8)}$. En estos casos, la medida de evaluación de los beneficios es común a ambas alternativas y se elige los cocientes más bajos que representan las intervenciones más eficientes (Figura 2).

En los ACE se pueden comparar los efectos de las distintas opciones en unidades clínicas naturales, entre las cuales se encuentran, por ejemplo, los años de vida ganados, los ingresos hospitalarios evitados, el número de casos prevenidos, etc.
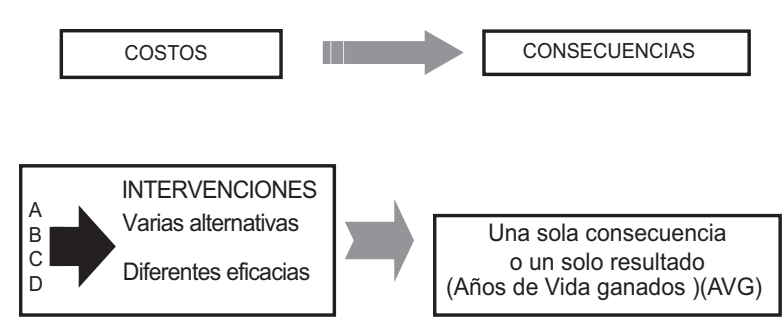

Figura 2. Esquema de análisis de costo-efectividad.

Por ejemplo, si se compara la hemodiálisis frente al trasplante renal para tratar la enfermedad renal crónica, la hemodiálisis tiene un costo de USD 40000 por "año de vida ganado" (AVG) y el trasplante renal tiene un costo al tercer año de USD 10000 por AVG. En este ejemplo, los costos se miden en dólares y la efectividad en unidades naturales como AVG y, obviamente, podemos concluir que el trasplante renal es una intervención más costo-efectiva.

En el ejemplo de la Tabla 2, se comparan dos tratamientos preventivos para evitar infartos. Se puede observar que la intervención $B$ tuvo un costo adicional de USD 25000 y la diferencia de efectos es de cinco infartos. La razón de costo-efectividad incremental (RCEI) es igual a USD 5000 por infarto. El tratamiento B es más costoso que el tratamiento $A$ en términos individuales, pero también generó ahorros debido a los infartos evitados. Al usar la intervención B es necesario invertir USD 5000 para evitar un infarto (beneficio sanitario neto). También podríamos calcular que evitar cinco infartos en esta población representa un beneficio de doce años de vida ganados. La razón de costo-efectividad también podría ser expresada como USD 2083 por AVG (USD 25 000/12).

\section{ANÁLISIS DE MINIMIZACIÓN DE COSTOS (AMC)}

EI AMC se utiliza cuando se comparan dos intervenciones en las que estudios previos han demostrado igual eficacia y efectividad. Se considera los menores costos que generan diferentes alternativas, cuyos resultados deben ser idénticos ${ }^{(11)}$. Por ejemplo, para una colecistectomía laparoscópica se requiere de la tecnología y personal adiestrado que maneje el equipo (costos); mientras que una colecistectomía convencional a cielo

Tabla 2. Resultados del análisis de costo-efectividad de dos intervenciones.

\begin{tabular}{ccccccc}
\hline Intervención & N. ${ }^{\circ}$ pacientes & Costos (USD) & N. ${ }^{\circ}$ infartos & $\begin{array}{c}\text { Diferencias de } \\
\text { costos }\end{array}$ & $\begin{array}{c}\text { Diferencias de } \\
\text { efectos }\end{array}$ & RCEI $^{\star}$ \\
\hline A & 100 & 98000 & 15 & & & \\
B & 100 & 123000 & 10 & 25000 & 5 & 5000 \\
\hline
\end{tabular}

${ }^{*}$ RCEI (Razón Costo Efectividad Incremental): diferencia de costos /diferencia de efectos. 
abierto, parece ser más barata pero puede tener costos mayores por los riesgos quirúrgico-anestésicos y días de hospitalización. En estos estudios, ambas alternativas producen el mismo resultado y no tienen diferencias en cuanto a su efectividad. El objetivo principal de los AMC es encontrar la alternativa menos costosa, dado que las consecuencias son las mismas. Además, estos estudios permiten identificar la distribución de los costos y explicar por qué una alternativa resulta menos costosa.

\section{ANÁLISIS DE COSTO UTILIDAD (ACU)}

Los ACU son hoy en día el tipo preferido de EE, siendo una combinación de la evaluación de los años vividos y la calidad de vida. El concepto de "utilidad", tal como lo utilizamos en este artículo, se refiere a una valoración que hace la persona que disfruta (o sufre) un servicio o un estado determinado. En este tipo de estudios se utilizan "utilidades" que miden el sufrimiento que la enfermedad provoca en las personas. Desde esta perspectiva, el paciente es incorporado en la evaluación de la atención de salud. La utilidad derivada por el paciente de un estado determinado es también reflejada en la valoración cualitativa de tal estado, en la llamada "calidad de vida" y las mediciones de esta se asumen como mediciones de utilidad. Un aspecto importante a considerar es que la calidad de vida depende de muchos otros factores además de la salud, por lo cual algunos investigadores prefieren usar en estos casos el término "calidad de vida relacionada con la salud" ${ }^{(12,13)}$.

Los ACU buscan valorar la vida ganada no solo en términos de cantidad (años de vida), sino también de calidad. Las unidades obtenidas son los años de vida ajustados por calidad (AVAC) o QALY (Quality-adjusted life years), que es una medida de carga de enfermedad. Los resultados se presentan de la misma forma que en el análisis de costo efectividad, pero usando como denominador los AVAC obtenidos en lugar de la efectividad (Figura 3).

Otra medida de la carga de enfermedad son los años de vida ajustados por discapacidad (AVAD) o DALY (Disability adjusted life years), que se expresan como el número de años perdidos debido a la enfermedad, discapacidad o muerte prematura.

Los datos sobre esperanza de vida sana (o la esperanza de vida libre de discapacidad) indica el número de años que una persona de una determinada edad puede esperar vivir sin discapacidad (EVLD - expectativa de vida libre de discapacidad) ${ }^{(14,15)}$.

Las principales razones para utilizar este tipo de análisis son: i) Cuando las intervenciones tienen múltiples

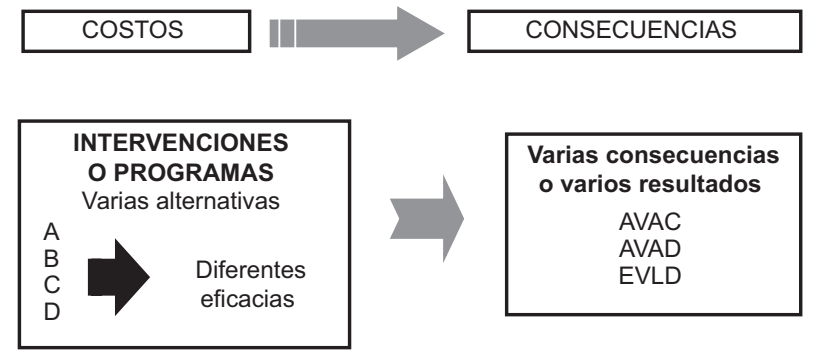

Figura 3. Esquema de los análisis de costo-utilidad.

consecuencias y todas nos interesan, por ejemplo en el tratamiento del sedentarismo con el entrenamiento físico podemos evaluar el peso perdido, eventos cardiovasculares, mejora de la dislipidemia, disminución de la depresión y la ansiedad y ii) cuando nos interesa obtener un resultado que permita comparar alternativas que no se relacionan entre sí y no comparten las consecuencias, por ejemplo si queremos comparar la tasa de costo-utilidad del tratamiento de la desnutrición con el tratamiento del infarto (intervenciones no relacionadas) y estamos interesados en medir; la sobrevida y la calidad de vida que cada intervención conlleva.

La ventaja del ACU es que permiten resumir todos los efectos de las intervenciones en un único valor; el AVAC, y utilizar este valor para compararlo con otro tratamiento que, a su vez, puede tener múltiples efectos, incluso diferentes.

El AVAC es una medida que intenta expresar a cuanto equivale un año viviendo con una determinada condición. La "unidad" utilizada para esta comparación es el año vivido en plena salud. Por ejemplo, si decimos que el AVAC de la diálisis es de 0,55; lo que estamos diciendo es que vivir un año en diálisis equivale a vivir 0,55 años en plena salud. Esto es debido a que la diálisis afecta la calidad de vida, por eso un año en diálisis "vale" menos que un año sin diálisis

En la Tabla 3; se compara dos tratamientos para el cáncer de próstata, uno de los ello prolonga la vida pero con ciertos efectos adversos (como incontinencia urinaria o impotencia). Si comparamos las intervenciones como un ACE la intervención $B$ tendría un costo adicional de USD 30 000. La diferencia de efecto será de USD 30000 por año de vida ganado.

En este primer resultado solo tuvimos en cuenta un resultado: la prolongación de la vida. No tuvimos en cuenta las diferencias en calidad de vida entre ambas intervenciones. Para tener en cuenta ambos factores debemos utilizar los AVAC. Como vemos, el tratamiento A logra una sobrevida de cuatro años con una calidad de vida equivalente a 0,95 AVAC. Por lo tanto, estos cuatro años de 
Tabla 3. Resultados del análisis de costo-utilidad de dos intervenciones.

\begin{tabular}{ccccccccc}
\hline Intervención & $\begin{array}{c}\mathbf{N}^{\circ} \\
\text { pacientes }\end{array}$ & $\begin{array}{c}\text { Costos } \\
\text { (USD) }\end{array}$ & $\begin{array}{c}\text { Diferencia de } \\
\text { costos (USD) }\end{array}$ & AVG & $\begin{array}{c}\text { Calidad de } \\
\text { vida (AVAC) }\end{array}$ & AVAC & $\begin{array}{c}\text { Diferencia } \\
\text { de efectos }\end{array}$ & RCUI * \\
\hline A & 100 & 45000 & & 4 & 0,95 & 3,8 & & \\
B & 100 & 75000 & 30000 & 5 & 0,80 & 4,0 & 0,2 & 150000 \\
\hline
\end{tabular}

${ }^{*}$ RCUI (Razón Costo-Utilidad incremental): diferencia de costos/diferencia de efectos.

sobrevida equivalen a 3,8 años en plena salud o AVAC $(4 \times 0,95)$. El tratamiento $B$ logra una sobrevida de cinco años y una calidad de vida menor (0,80 AVAC). Los cinco años de sobrevida equivalen a cuatro años en plena salud o AVAC.

Un ACU utilizará esta medida para calcular la tasa o razón de costo-utilidad. Desde esta perspectiva, la diferencia ahora entre ambas intervenciones ya no es un año de vida sino 0,2 AVAC $(4,0-3,8)$.

Los resultados de los ACU se expresan como un cociente; la "razón costo-utilidad incremental" (RCUI). En el ejemplo, si tenemos las diferencias de costos (USD 30 000) y la diferencias de efectos (0,2 AVAC), entonces la TCUI será: 30000 / 0,2 que es igual a USD 150000 por AVAC. Es más evidente ahora que la diferencia entre ambas intervenciones no es tan importante como parecía en un inicio, ya que la prolongación de la vida en un caso se ve compensada por la mejora de la calidad de vida en el otro.

Otro aspecto importante en los ACU es la obtención de Ios AVAC, para conocer el "valor" o "utilidad" que una persona le da a un determinado estado de salud; podríamos emplear el siguiente método: A un paciente en diálisis le preguntamos por cuánto tiempo de salud plena estaría dispuesto a cambiar un año en diálisis. Supongamos que esta persona contesta que vivir un año en diálisis le resultaría equivalente a vivir ocho meses en plena salud. Con esta información podríamos estimar cual es la "equivalencia" para esta persona: ocho meses en plena salud $=12$ meses en diálisis; entonces 8 / 12 = 0,67 AVAC. Para esta persona, un año en diálisis equivale a 0,67 años en plena salud. Esta misma evaluación podríamos hacerla a varias personas representativas de una población y obtener un valor promedio para este AVAC. Este método de cálculo de AVAC, es el método de "compensación temporal" o Time Trade Off, debido a que se basa en estimar cuánto tiempo de vida está dispuesto a sacrificar una persona para pasar a plena salud ${ }^{(16)}$.

\section{DESTINATARIOS O USUARIOS}

Es importante tener en cuenta al realizar una EE quiénes serán los destinatarios de ella, es decir los usuarios. En principio, son todas las personas encargadas de la toma de decisiones para las cuales la información de la evaluación resulte útil. Esta audiencia puede ser muy distinta de aquellos que realizaron la investigación. Pueden ser gobiernos, ministerios de salud, hospitales, entes financiadores de salud, etc. Es muy importante identificar y conocer la audiencia de la EE en el diseño del estudio, para decidir los métodos más adecuados y la forma de presentar los resultados.

\section{PERSPECTIVA O PUNTO DE VISTA}

Se refiere al escenario en el que nos colocaremos para evaluar el problema. No es lo mismo si usamos la perspectiva del presidente de un país o del Ministerio de Economía, que si lo hacemos desde la perspectiva del director de un seguro privado de salud. Es fundamental definirla desde el diseño del estudio, ya que determina qué tipo de costos son necesarios considerar. Algunas perspectivas son del paciente, del médico, del hospital, del financiador y de la sociedad. La gran mayoría de las EE se realizan desde el punto de vista del financiador, aunque cada vez más se tiende a contemplar la perspectiva social ${ }^{(17)}$.

\section{HORIZONTE TEMPORAL}

Al comparar dos o más tecnologías para algún problema sanitario es importante definir cuál será el horizonte temporal para esta evaluación. Por ejemplo, al comparar drogas antihipertensivas podemos evaluar un horizonte temporal corto (un mes o tres meses) o también uno largo (cuánto altera la sobrevida y la calidad de vida de los pacientes). Existe un horizonte de toda la vida o el horizonte según la expectativa de vida de un país, para el caso de personas saludables.

\section{DESCUENTOS}

El descuento es el proceso de calcular cuál es el valor actual de algo que sucederá. Entonces, el valor presente neto (o valor actual) de algo que sucederá, depende de cuán lejos vaya a suceder, así como de la fuerza de esa preferencia temporal, reflejada en la tasa a la cual se descuente (tasa de descuento). Muchos estudios también concluyen que existe una preferencia temporal por los beneficios sanitarios (AVG, AVAC). Es por ello que en las evaluaciones económicas no solo es importante calcular cuál es el valor presente neto (VPN) de los costos, sino también calcular el VPN de los beneficios 
de cada estrategia. Si una medicación cuesta USD 100 cada año de tratamiento, el gasto en el año número 20 no es el mismo que tenemos que gastar hoy. A esta característica se llama preferencia temporal por el dinero. Las EE no solamente evalúan los costos y beneficios totales que implican las diferentes tecnologías, sino también en qué momento del horizonte temporal ocurren estos costos y sus resultados.

La recomendación actual es que se use la misma tasa de descuento tanto para los costos como para los beneficios. Las tasas recomendadas pueden variar de país a país, siendo usual del $3 \%$ (recomendada por el Panel de Washington), y en el caso de países de nuestra región Brasil y México, recomiendan usar una tasa del $5 \%$.

El descuento es "el revés" de las tasas de interés de los bancos. Si se invierte USD 100 a una tasa de interés $(r)$ del $3 \%$, en un año se obtendrá USD 103. El descuento es el reverso de este proceso de intereses, y se utiliza para calcular el VPN de los USD 100 que serán gastados en el año próximo. La cantidad de dinero que me dará USD 100 el año próximo (a una tasa del 3 \%) será USD 0,97 (VPN = USD 100/1,03 = USD 97).

La siguiente es la fórmula general para calcular el valor actual de algo que sucederá (en dinero o años): VPN $=$ Valor Futuro $/(1+r) t$. Donde $r$ : tasa de descuento expresada en fracción decimal $(E \mathrm{j} .0,03)$. $t$ : tiempo (año) $y(1+r) t$ : factor de descuento ${ }^{(10)}$.

\section{PRINCIPALES VEHÍCULOS DE LAS EVALUACIONES ECONÓMICAS}

La forma como se realizan en la vida real las EE son dos:

i) Evaluaciones económicas basadas en datos de pacientes individuales. Básicamente se obtienen datos clínicos y económicos de los ensayos clínicos u de otros estudios (revisiones sistemáticas y metanálisis), estas EE se efectúan dentro de un ensayo clínico (piggy-back).

ii) Evaluaciones económicas basadas en modelos. En este caso se construye un modelo que sintetiza la información de distintas fuentes para evaluar el costo-efectividad de las intervenciones. Estos modelos son: a) árboles de decisiones simples y recursivos; b) modelos de simulación epidemiológica; c) modelos de Markov; y d) simulación de eventos discretos u otros modelos.

\section{EVALUACIÓN DE LA INCERTIDUMBRE}

La potencia de un modelo de EE se puede evaluar a través del "análisis de sensibilidad". Con estas técnicas se evalúan cuánto afecta la incertidumbre de los resultados de los modelos. Se puede hacer análisis de sensibilidad de una vía, donde se evalúa una sola variable; por ejemplo, tomando los valores o los intervalos de una tasa de prevalencia se puede analizar cuánto varían los resultados y eventualmente poder variar las conclusiones.

\section{REGLAS DE DECISIÓN}

El resultado principal de una evaluación económica se puede expresar en la razón o tasa de costo- efectividad. Esta se puede visualizar en un plano de costo-efectividad, que compara las nuevas tecnologías contra la tecnología actual utilizada, tanto en relación a la diferencia de costos y de efectos (Figura 4).

Si observamos el cuadrante superior izquierdo (la nueva tecnología es más costosa y menos efectiva, o dominada), o inferior derecho (la nueva tecnología es más efectiva y menos costosa, o dominante) no tiene sentido calcular la tasa de costo-efectividad incremental (TCEI) para tomar la decisión correcta de rechazar o adoptar la nueva tecnología. Lamentablemente, las nuevas tecnologías "caen" generalmente en un cuadrante más conflictivo: son mejores, pero también más costosas (superior derecho).

\section{EVALUACIÓNDELOSBENEFICIOS DEL TRATAMIENTO EN RELACIÓN A LOS DAÑOS Y LOS COSTOS}

Un modo simple de clasificar los resultados de evaluaciones económicas que expresen sus resultados en costos y efectos incrementales; es una matriz de $3 \times 3$ con nueve celdas que clasifican los estudios según si el nuevo tratamiento es más, igual, o menos costoso que el comparado y también si su efectividad es mayor, igual o peor (Figura 5).

En la categoría 1, el tratamiento es menos costoso y más efectivo, se dice que el nuevo tratamiento tiene dominancia fuerte por lo que se acepta el nuevo tratamiento. De manera inversa, la categoría 2 representa dominancia fuerte para rechazar el nuevo tratamiento, ya que resulta más costoso y menos efectivo.

Existen cuatro casos de los denominados "dominancia débil" donde los costos o la efectividad son similares entre los dos tratamientos: la categoría 3 indica dominancia débil para aceptar el tratamiento (costo equivalente pero más efectivo) y la categoría 4 indica dominancia débil para rechazar el tratamiento (mayor costo con similar efectividad). De manera análoga, las categorías 5 y 6 indican dominancia débil para rechazar y aceptar el tratamiento, respectivamente. 


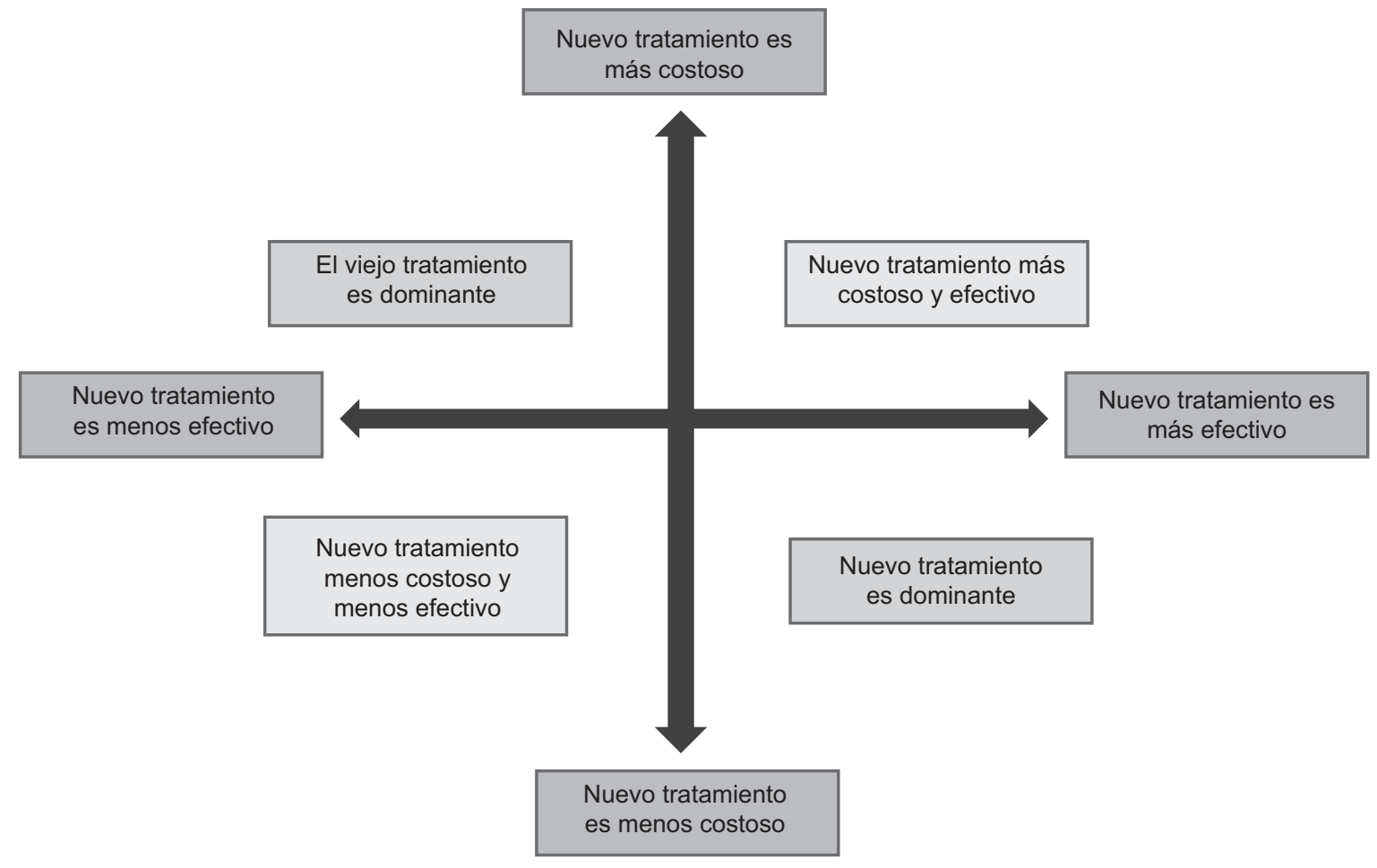

Figura 4. Plano de costo-efectividad.

En las seis categorías en las cuales hay dominancia fuerte o débil, no hace falta realizar ningún análisis adicional para ayudar a la toma de decisiones. Son las categorías 7 a 9 donde no hay dominancia y es necesario avanzar más en el análisis. La más frecuente de las tres es la categoría 7, en las que el nuevo tratamiento ofrece un beneficio adicional pero a un costo mayor (por ejemplo el uso del fármaco trombolítico rt-PA frente a la estreptoquinasa). En estas circunstancias es útil el cálculo de la tasa de costo-efectividad incremental de los nuevos tratamientos. Luego de estimar las tasas de costo-efectividad y, suponiendo que es aplicable a nuestra realidad, ¿cómo decidimos el valor del precio a pagar por salvar un año adicional de vida en la población? Los resultados hay que cotejarlos con algún criterio externo para evaluar si el hospital o la sociedad están dispuestos a pagar este precio para lograr esta mejoría en la población a su cargo. Si existen varias estrategias que

\section{Costo incremental del tratamiento comparado con el control}

\section{Efectividad del tratamiento comparado con el control}

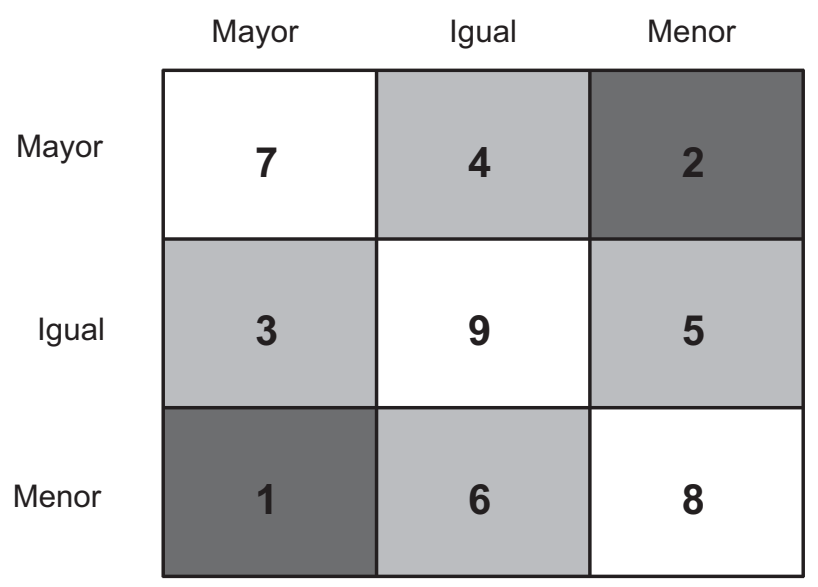

Figura 5. Matriz de nueve posibles resultados de un estudio de costo-efectividad. 
tienen una costo-efectividad favorable y comparable, ¿cómo tomar la decisión?, muchas veces se utiliza un umbral, muy controvertido pero que refleja hasta cuánto está dispuesta la sociedad a pagar por un año de vida. Uno aceptable y muy utilizado en la literatura es el de USD 50000 por año de vida ganado, dato desconocido en el Perú debido a la escasez de EE locales ${ }^{(18)}$.

\section{TRANSFERIBILIDAD Y GENERALIZACIÓN DE LAS EVALUACIONES ECONÓMICAS}

La gran mayoría de las intervenciones que utilizamos en nuestros países, desde vacunas hasta tratamientos para el cáncer, se basen en estudios de eficacia provenientes de otros ámbitos. Esto es así debido a que existe un amplio consenso que el efecto relativo de las intervenciones "viaja" fácilmente de un ámbito a otro. Pero si estamos ante una evaluación económica realizada en Reino Unido ¿podemos aplicarla directamente en Brasil, México o Perú? La respuesta en realidad es no.

Se ha definido la generalización como "el grado en el que los resultados de un estudio, conducido en una población de pacientes en un contexto específico, es aplicable y válido en otra población en un contexto diferente sin ningún tipo de adaptación o ajuste" ${ }^{(19)}$.

Mientras, que Drummond ha definido la transferibilidad como "el potencial de adaptar o ajustar el análisis del estudio para hacer que los resultados sean relevantes en diferentes ámbitos".

Son diversos los factores que hacen que la costo-efectividad de las tecnologías varíe de un lugar a otro. Los cuales se pueden agrupar en aspectos relacionados al comportamiento del problema de salud en el ámbito local (por ejemplo, la incidencia o la gravedad del problema); la disponibilidad de recursos sanitarios y los tratamientos disponibles para tratarlo; las prácticas de tratamiento locales (en USA tienen prácticas más "invasivas" para manejar la enfermedad coronaria que los países de Europa); y en las diferencia de costos (en la mayoría de los países de nuestra región, los costos del tiempo médico o de internación, son relativamente menores que en USA u otros países desarrollados, mientras que los costos de las nuevas tecnologías son muchas veces similares).

Augustovski evaluó la transferabilidad de EE en Latinoamérica, y observó que en general era muy difícil trasladar resultados de un ámbito a otro, debido a que la gran mayoría de los estudios presentaban problemas metodológicos o de informes que impedían la transferibilidad a otros ámbitos ${ }^{(20)}$.

\section{CONCLUSIONES}

En este artículo hemos introducido los conceptos iniciales de las EE como herramienta que asiste a la toma de decisiones. Con estos elementos esperamos que los lectores puedan comprender y aplicar los resultados de una EE en las tomas de decisiones ya sea en su práctica clínica o como decisores en políticas de salud.

\section{Conflictos de interés}

Los autores declaran no tener conflictos de interés en la publicación de este artículo.

\section{Fuente de financiamiento}

Autofinanciado.

\section{REFERENCIAS BIBLIOGRÁFICAS}

1. Rodríguez-Ledesma MA, Vidal-Rodríguez C. Conceptos básicos de economía dela salud para el médico general. Rev Med Inst Mex Seguro Soc. 2007;45(5):523-32.

2. Del Prete S. Economía y salud en tiempos de reformas. Buenos Aires: Editorial. Universidad Nacional de La Plata; 2000.

3. Taylor RS, Drummond MF, Salkeld G, Sullivan SD. Inclusion of cost effectiveness in licensing requirements of new drugs: the fourth hurdle. BMJ. 2004;329:972-75.

4. Normand C, Bowling A. A cost-benefit analysis. In: Swash M, Wilden J (Eds). Outcomes in Neurological and Neurosurgical Disorders. Cambridge, Cambridge University Press, 1998.

5. Collazo HM,Cárdenas JR,González RL,Miyar RA,Gálvez AM, Cosme JC. La economía del la salud: ¿debe ser de interés para el campo sanitario?.Rev Panam Salud Publica. 2002;12(5):359-65.

6. Donaldson $\mathbf{C}$. The state of the art of costing health care for economic evaluation. Community Health Studies 1990;14(4):341-56.

7. Puig-Junoy J, Ortún-Rubio V, Pinto-Prades JL. Los costes en la evaluación económica de tecnologías sanitarias. Aten Primaria. 2001;27(3):186-9.

8. Ament A, Evers S.Cost of illnes studies in health care: a comparison of two cases. Health Policy.1993;26(1):29-42.

9. Puig-Junoy J, Pinto-Prades JL, Ortún-Rubio V. El análisis coste-beneficio en sanidad. Aten Primaria. 2001;27(6):422-7.

10. Folland S, Goodman AC, Stano M. Cost-Benefit. Analysis and Other Tools of Economic Evaluation. In: The Economics of Health and Health care. 3ra ed. Upper Saddle River, NJ: Prentice-Hall; 2001. p. 74-93.

11. Thornton J. Health economics: a beginner's guide. British J Hosp Med. 1997;58(11):547-50.

12. Guyatt GH, Van Zanten SJOV, Feeny DH, Patrick DL. Measuring Quality of Life in Clinical Trials: A Taxonomy and Review. CMAJ. 1989;140:144-8.

13. Guyatt GH, Feeny DH, Patrick DL. Measuring Health related Quality of Life. Ann Intern Med. 1993;118(8):622-9. 
14. Drummond M, Stoddart G, Labelle R, Cushman R. Health economics: An introduction for clinicians. Ann Intern Med. 1987;107(1):88-92.

15. Eisemberg J. Clinical economics. A guide to the economic analysis of clinical practices. JAMA. 1989;262(20):2879-86.

16. Pichon-Riviere A, Augustovski F. Introducción a las Evaluaciones Económicas en Evaluación de Tecnologías sanitarias. Documento Educativo. Buenos Aires: IECS.

17. Meltzer MI. Introduction to Health Economics for Physicians. The Lancet. 2001;358:993-8.

18. Augustovski F. Evaluaciones Económicas en Salud II. Evidencia. 2002;5(5):154-7.

19. Sculpher MJ, Pang FS, Manca A, Drummond MF, Golder $\mathrm{S}$, Urdahl $\mathrm{H}$, et al. Generalisability in economic evaluation studies in healthcare: a review and case studies. Health Technol Assess. 2004;8(49):III-IV, 1-19
20. Augustovski F, Iglesias C, Manca A, Drummond M, Rubinstein A, Martí SG. Barriers to Generalizability of Health Economic Evaluations in Latin America and the Caribbean Region. Pharmacoeconomics. 2009;27(11):919-29.

Correspondencia:César Antonio Loza Munarriz

Facultad de Medicina Alberto Hurtado, Universidad Peruana Cayetano Heredia. Lima, Perú.

Dirección: Pasaje Santiago 2236 Jesús María. Lima, Perú.

Teléfono: (511) 462-2024

Correo electrónico: cloza44@gmail.com; cesar.loza@upch.pe

\section{Suscríbase en forma electrónica y gratuita a los contenidos de la Revista Peruana de Medicina Experimental y Salud Pública, ingrese a WWW.ins.gob.pe, seleccione el ícono de la revista y envíenos sus datos.}

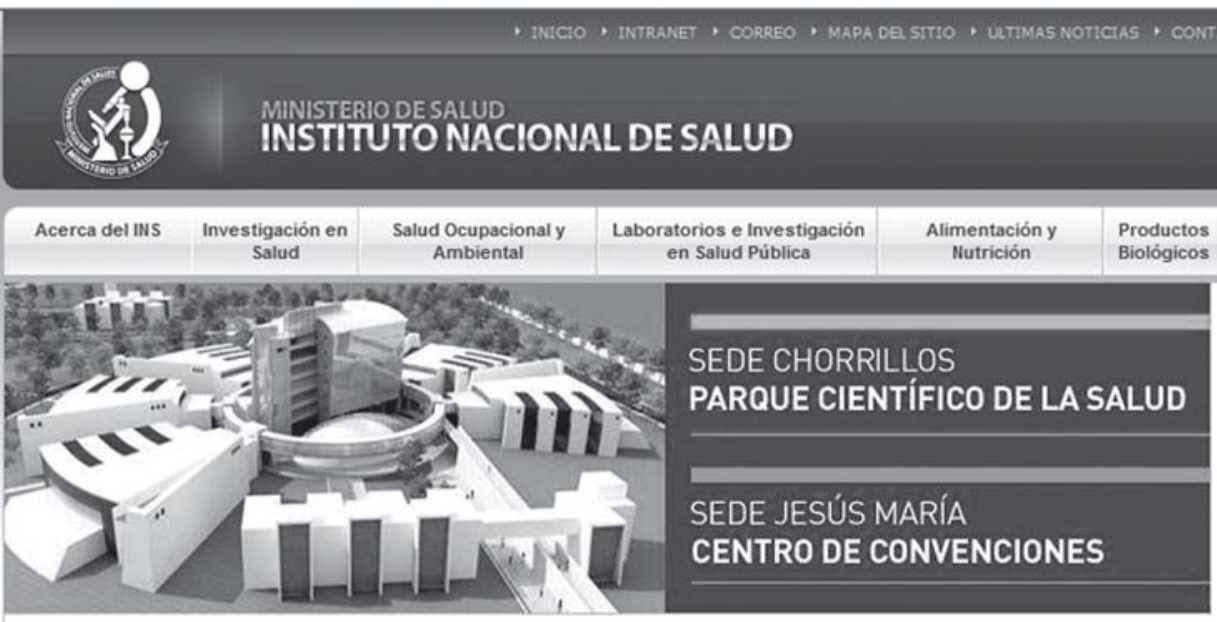

ÚLTIMAS INVESTIGACIONES Y EVIDENCIAS CIENTÍFICAS

Ver Todas

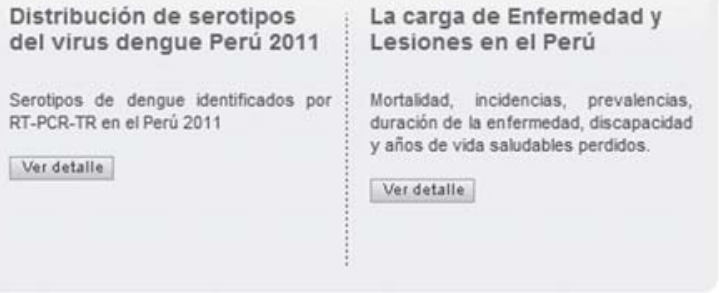

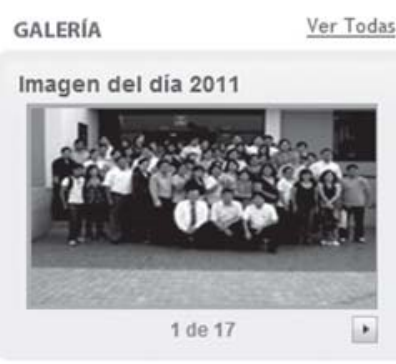

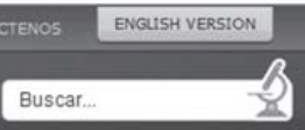

\begin{tabular}{|l|l|} 
Salud & Control de Calidad
\end{tabular} Intercultural de Medicamentos

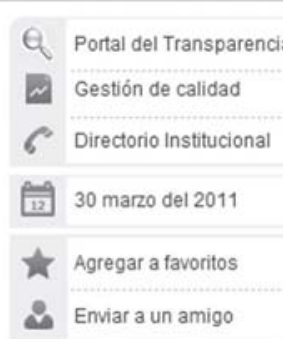

Publicaciones

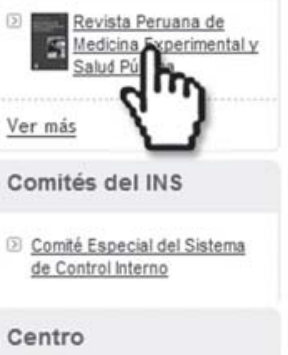

